\title{
3D Freehand Echocardiography for Automatic Left Ventricle Reconstruction and Analysis Based on Multiple Acoustic Windows
}

\author{
Xujiong Ye, J. Alison Noble, and Jerome Declerck \\ Medical Vision Laboratory, Department of Engineering Science, University of Oxford, \\ Parks Road, Oxford OX1 3PJ, UK. \\ \{xujiong, noble, jdecler\}@robots.ox.ac.uk
}

\begin{abstract}
A new method is proposed to reconstruct and analyse the left ventricle (LV) from multiple acoustic windows 3D ultrasound acquired using a 3D rotational probe. Prior research in this area has been based on one acoustic window acquisition. However, the data suffers from several limitations that degrade the $3 \mathrm{D}$ reconstruction, such as motion of the probe during the acquisition and the presence of shadow due to bone (ribs) and air (in the lungs). In this paper we aim to overcome these limitations by automatically fusing information from multiple acoustic windows sparse-view acquisitions and using a position sensor to track the probe in real time. Geometric constraints of the object shape, and spatio-temporal information relating to the image acquisition process are used to propose new algorithms for (1) grouping endocardial edge cues from an initial image segmentation and (2) defining a novel reconstruction method that utilises information from multiple acoustic windows. We illustrate our new method on phantom and real heart data and compare its performance against our previous approach that is based on a single acoustic window.
\end{abstract}

\section{Introduction}

Advances in both acquisition technology and computing power have meant that three dimensional (3D) cardiac image analysis has become an active area of research. In particular, 3D cardiac ultrasound offers potential advantages over X-ray angiography and nuclear medicine methods in terms of its lack of ionising radiation and, with respect to cardiac MR, better spatial resolution.

The state-of-the-art method for 3D cardiac ultrasound acquisition uses a 3D rotational scanning configuration, in which the transducer rotates about a central axis of the probe at a fixed angle increment. This generates a series of $2 \mathrm{D}$ sequences with a common axis of rotation for different cardiac cycles.

Most prior research on 3D ultrasound left ventricle (LV) analysis has focused on one acoustic window [1][2][3]. However, in this case, the data suffers from several limitations that degrade the reconstruction and reduce the clinical value. Most notably: 1) The presence of bone (ribs) and air (in the lungs) makes integration of 2D slices from one acoustic window particularly difficult, as large parts of the field of view are shadowed. 2) Respiration gating is employed to obtain "optimal" image 
acquisition quality. The net effect is that acquisition times can be long (several miniutes) and this inevitably results in slight motion of the probe during the scanning.

In this paper we aim to overcome these limitations by using a position sensor to track the probe in real time and fusing automatically detected image features from multiple acoustic window sparse-view acquisitions. We use geometric constraints of the object shape and image acquisition process to propose new algorithms for (1) grouping endocardial edge cues from an initial image segmentation and (2) defining a novel reconstruction method that utilises information from multiple acoustic windows. We illustrate our new method on phantom and real heart data and compare its performance to our previous approach that was based on a single acoustic window.

The most closely related research to our own is the work by Legget et al [4]. The major differences are that they used 2D scanning rather than $3 \mathrm{D}$ rotational scanning and $3 \mathrm{D}$ reconstruction was based on manually delineated feature points.

\section{Proposed Method and Algorithms}

Figure 1 shows a flow-diagram outlining the key steps in our approach. In the following section each step is described.

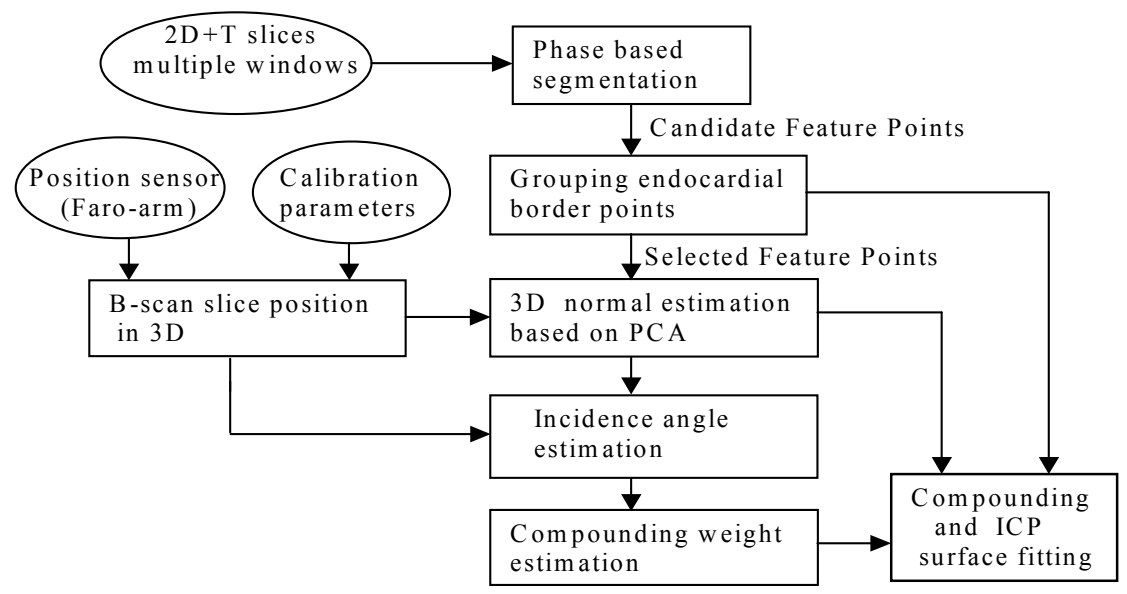

Fig. 1. Overall block diagram of the proposed method.

\subsection{Image Acquisition}

Digital 3D+T data was acquired on a HP SONOS 5500 ultrasound machine (Agilent Technologies) using a 3-5MHZ 3D rotating transducer with the Faro-arm position sensor (FARO Technologies) attached to enable real-time recording of probe position. The angle increment of the rotational probe was set to 6 degrees, so that for each acoustic window 30 co-axial slices were obtained. The Faro-arm was calibrated using an in-house calibration routine based on a ping-pong ball calibration object [5]. 
Scanning was performed using ECG and respiration gating on two acoustic windows: apical long-axis view and parasternal short-axis view. Subjects were asked to remain as still as possible during data acquisition so that there was a good spatial alignment between the different views. Data was acquired at a rate of 25 frames per second.

\subsection{Phase Congruency Based Segmentation}

A phase congruency (PC) based feature detection method is used to find candidate endocardial points [6]. Briefly, this algorithm involves calculating a measure of feature asymmetry (edgeness) using a band of log-Gabor wavelet filter pairs spread in a $2 \mathrm{D}$ or $3 \mathrm{D}$ space. The measure values vary from a maximum of 1 , indicating a very significant feature, down to 0 , indicating no significance. This provides an intensity invariant feature detection method. The normal to the features can also be estimated and the direction of the normal is defined as a negative edge (from bright to dark).

This segmentation method provides accurate localisation of endocardial borders in situations of changing image contrast and poor signal-to-noise ratio. But one of the disadvantages of this method is that it produces a large number of "spurious" noisy points at the same time (Figure 3(a)). To reject these points, the following grouping method has been developed which exploits spatio-temporal information and geometric knowledge of the LV.

\subsection{Grouping Endocardial Border Points}

\subsubsection{Selection of Geometrically Consistent Points}

Suppose there are two candidate boundary points $a$ and $b$ in a 2D image, with corresponding normals $\vec{n}_{a}$ and $\vec{n}_{b}$ (Figure 2). We let:

$$
f_{1}=\frac{\vec{c}_{a b} \cdot \vec{n}_{a}}{\left\|\vec{c}_{a b}\right\| \cdot\left\|\vec{n}_{a}\right\|} \quad, \quad f_{2}=\frac{\vec{c}_{b a} \cdot \vec{n}_{b}}{\left\|\vec{c}_{b a}\right\| \cdot\left\|\vec{n}_{b}\right\|}
$$

where $\vec{c}_{a b}$ is a distance vector from point $a$ to point $b$. i.e, $\vec{c}_{a b}=b-a$.

During a complete cardiac cycle, the LV basically appears as a cylinder in a $2 \mathrm{D}+\mathrm{T}$ data set. Given this geometric assumption, both $a$ and $b$ are

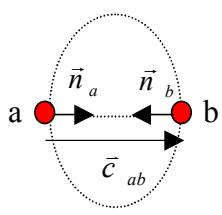

Fig.2. A definition of face-to-faceness for two points. defined to be "interesting" boundary points if they satisfy the following three criteria:

(1) The endocardial boundary points must have sufficient face-to-faceness [7] $F$ defined as $F(a, b)=f_{1} \cdot f_{2}$. Values for $F$ near +1 occur when both boundary points face inward (or outward). An error threshold $\varepsilon$ is set for acceptable face-tofaceness, namely, $F(a, b)>1-\varepsilon$.

(2) The normals of endocardial points are inward, namely, $f_{1}>0$ and $f_{2}>0$.

(3) The distance from one boundary point to the other must lie between $c_{\min }$ and $c_{\max }$, the parameters are determined by the structure of $\mathrm{LV}$. 
Figure 3(b) shows the result of applying the above three criteria on an initial PC based segmentation end-diastole image (Figure 3(a)). A low error threshold for acceptable face-to-faceness was set $(\varepsilon=0.05)$. Referring to this figure, note that the output of this shows that most of the noisy points are filtered, but some spurious boundary points are still present. Further, some real boundary points have been filtered due to shadow on one part of image. To further recover the endocardial feature points and reject the spurious points the following refinement step is adopted.

\subsubsection{Refinement of Edge Points Grouping}

The refinement process involves fitting a cubic parametric B-spline curve to the selected candidate feature data points that is represented as:

$$
p(u)=\sum_{i=0}^{n} d_{i} B_{i, 3}(u) \quad, \quad u \in\left[u_{3}, u_{n+1}\right]
$$

where $d_{i}$ represents the $i$-th control point (of $n$ control points), $B$ is the B-spline basis function attached to the parameterisation $u$, defined on a regular knot distribution $u_{i}$.

There are two advantages in using this step. First, we can define more accurately region of interest (ROI) around the endocardial boundary. This is useful for automatically defining a processing ROI that can speed up the analysis on the following time frames, as the endocardial boundary on the following frames is inside the boundary region of the first time frame (end-diastole). Second, the normal at each point on the fitting curve can be calculated by its tangent vector that is defined as:

$$
p^{(1)}(u)=\sum_{i=0}^{n} d_{i}^{(1)} B_{i, 2}(u), \quad d_{i}^{(1)}=3 \cdot \frac{d_{i}-d_{i-1}}{u_{i+3}-u_{i}}
$$

here $p^{(1)}(u)$ is the first differential. The other parameters are the same as in Eq. (2).

Both the location and normal vector are considered in the distance measurement to recover the missing endocardial feature points and reject further spurious points. Figure 3(c) shows a fitting result on the selected feature points; Figure 3 (d) shows the final result of grouped endocardial boundary points.

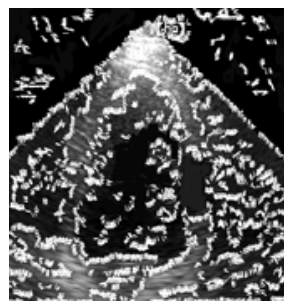

(a)

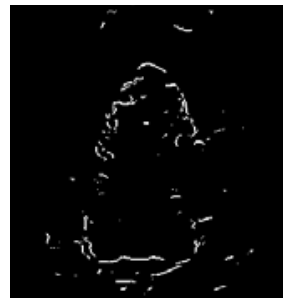

(b)

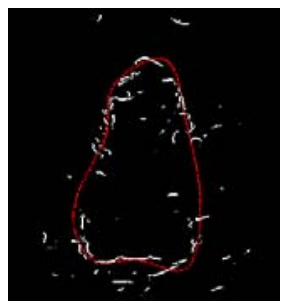

(c)

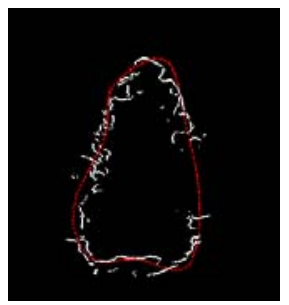

(d)

Fig. 3. (a) Initial PC based candidate boundary points overlaid on the end-diastole data slice. (b) Selection of endocardial feature points using face-to-faceness method. (c) A cubic B-spline fitting on feature points. (d) Final result of grouped endocardial features with the fitting curve. 


\subsection{D Reconstruction from Multiple Acoustic Windows}

We now turn to the problem of how to recover a 3D endocardial surface. The first step is to reconstruct a 3D data volume from a series of grouped endocardial feature points obtained from multiple acoustic windows. In our freehand imaging system the real 3D location of every feature point in every B-scan slice can be computed and combined into a 3D data volume after the calibration process. The "nearest neighbour" approach is used in this step, which means for each pixel, the nearest voxel is filled with the value of that pixel. For overlapping data from different views, rather than using the maximum or average intensity method, we adopt a weighted compounding approach in which the weight of each point is determined by the incidence angle of the ultrasound beam that is defined between the scanning beam and the $3 \mathrm{D}$ normal to the surface at the given point [8].

\subsubsection{Estimating the 3D Normal Based on Principal Components Analysis}

The $3 \mathrm{D}$ feature point normal can be estimated from the neighbouring data points using principal components analysis (PCA), which is described as follows:

For each 3D data point $x_{i}$, k points which are closest to $x_{i}$ are found. This set is denoted by $\operatorname{Nbhd}\left(x_{i}\right)$. Our purpose is to find a $3 \mathrm{D}$ vector $n_{i}$ that is most orthogonal to the k-neighbourhood set as a whole by solving the eigenvalue equation:

$$
R q=\lambda q
$$

where $R=\sum_{x \in \operatorname{Nbhd}\left(x_{i}\right)}\left(x-o_{i}\right) \otimes\left(x-o_{i}\right)$, and $o_{i}$ is the centroid of $\operatorname{Nbhd}\left(x_{i}\right)$.

If $\lambda_{i}^{1} \geq \lambda_{i}^{2} \geq \lambda_{i}^{3}$ denote the eigenvalues of $R$ associated with unit eigenvectors $\vec{q}_{i}^{1}$, $\vec{q}_{i}^{2}, \vec{q}_{i}^{3}$ respectively, $n_{i}$ is chosen to be either $\vec{q}_{i}^{3}$ or $-\vec{q}_{i}^{3}$. In our case, the selection of the correct sign is determined by using the point's $2 \mathrm{D}$ in-plane component normal $\vec{n}_{2 d}$, which is obtained by the PC based segmentation. If $\vec{n}_{2 d} \cdot \vec{q}_{i}^{3}>0, \vec{q}_{i}^{3}$ is chosen as the $3 \mathrm{D}$ normal of the point $x_{i}$, while $-\vec{q}_{i}^{3}$ is for the condition of $\vec{n}_{2 d} \cdot \vec{q}_{i}^{3}<0$.

\subsubsection{Estimating the Compounding Weight}

For overlapping data from the multiple views, we use a weighted compounding approach. The compounding weight $\alpha$ is defined as $\alpha=|\cos (\theta)|$, where $\theta$ is the incidence angle at each point that is defined as an angle between the scanning beam and the $3 \mathrm{D}$ normal to the surface at a given point. The position sensor provides information about the probe's direction, or the direction of the scanning beam. The smaller the incidence angle $\theta$ is, the bigger the weight $\alpha$ assigns to this point. Thus, this method reflects the geometric constraints of image acquisition, and favours regions for which the scanning beam is close to the normal and rejects data for which the scanning beam is far away from the normal.

In the final step, as in our previous work, an Iterative Closest Point (ICP) based surface fitting algorithm is used to recover the endocardial surface [9][10]. 


\section{Experimental Results}

\subsection{Study on Glove Phantom}

One finger of a latex glove was fully filled with tap water as a phantom, and securely fixed in a small box containing water.

Data sets were acquired from two acoustic windows on this phantom. Figure 4(a) is the feature points in 3D selected using our method for one acoustic window and for second window in (b). The incidence angle weighted 3D feature points reconstruction from two windows is shown in (c) and the results of the ICP based surface fitting on each window and combined windows are shown in (d), (e) and (f), respectively.

The phantom volume measurement was calculated to be $230 \mathrm{ml}$ for the true volume while $220.73 \mathrm{ml}$ for the fitting result from two windows (Figure (f)), $194.22 \mathrm{ml}$ and $165.63 \mathrm{ml}$ from each window (Figure (d) and (e)). This demonstrates the two views method has better performance for recovery object shape than from a single window.

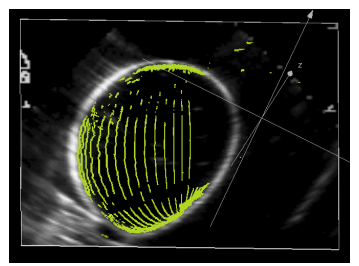

(a)

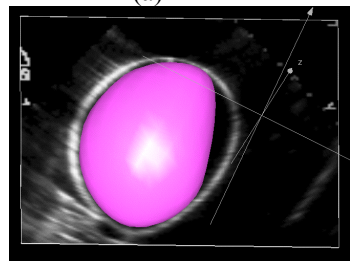

(d)

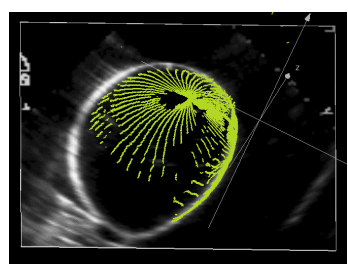

(b)

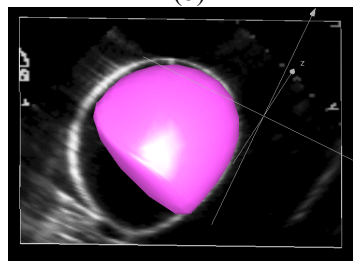

(e)

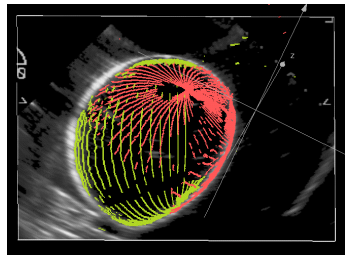

(c)

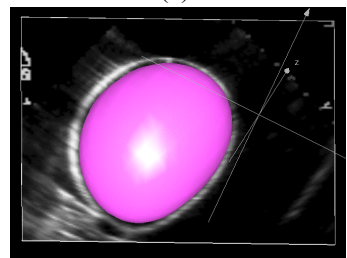

(f)

Fig. 4. Results on a glove phantom overlaid on one image slice. 3D grouped feature points on each acoustic window (a and b), and combined windows (c). ICP surface fitting on each window points ( $d$ and e) and combined window points (f).

\subsection{Study on Real Heart Data}

In this experiment, data sets were acquired from two acoustic windows: apical longaxis view and parasternal short-axis view. For each view (30 co-axial slices), scanning lasted 3-4 minutes during which there was slight motion of the probe. Figure 5 shows the true positions of the probe on each slice during two acoustic windows scanning. The maximum motion of the probe during each view acquisition is about $2 \mathrm{~mm}$. Figure 6 shows the 3D reconstruction of slices from two acoustic window acquisitions.

Figure 7 and Figure 8 show results using the new method. Figure 7 shows the projection of the 3D surfaces and grouped endocardial feature points onto one image slice. Figure 7(a) is from the apical long-axis view only, (a conventional view used in 


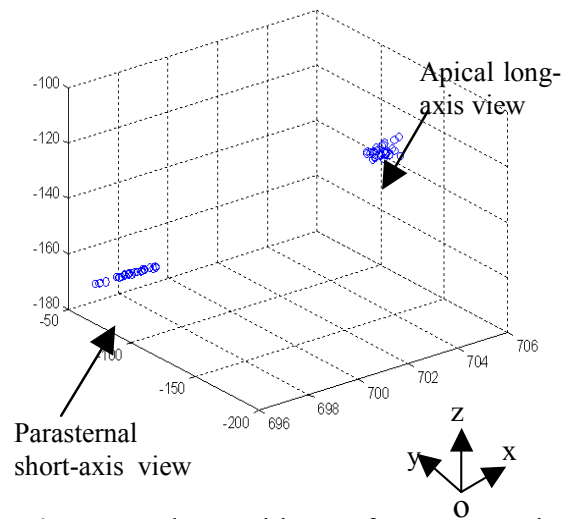

Fig. 5. Probe positions of two acoustic window scanning

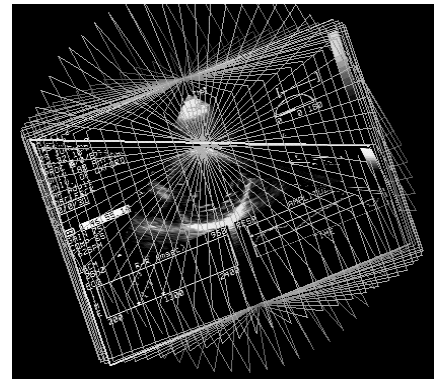

Fig. 6. 3D reconstruction of image slices from long-axis window and short-axis window acquisitions.
LV volume analysis from ultrasound), while (b) is to combine feature points from both the apical long-axis view and parasternal short-axis view. Note that, the gap between the grouped endocardial feature points in (a) can be connected using the feature points from the shortaxis view in (b).

In Figure 8, the 3D endocardial surfaces from the long-axis view, shortaxis view and combined views can be seen in (c), (d) and (e), respectively. Due to the large area of shadowing in the apical long-axis scanning, there is a big hole in the $3 \mathrm{D}$ features reconstruction

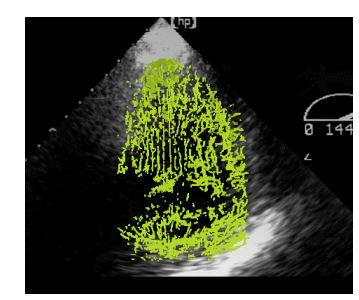

(a)
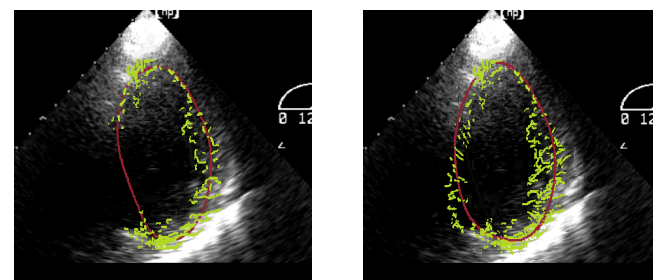

Fig. 7. Projection of the 3D fitted surface and 3D grouped endocardical feature points, lying within a clipped slab ( $2 \mathrm{~mm}$ width) centred on one image slice. Surface fitting on one apical endocardial boundary points (left) and on combined apical and parasternal endocardial boundary points (right).

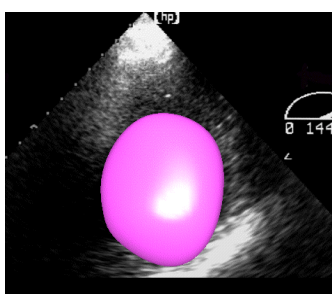

(d)

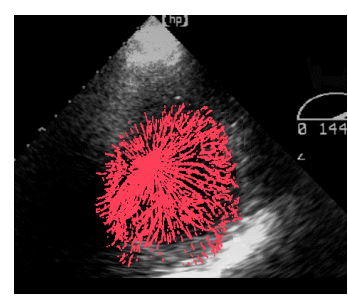

(b) (c)

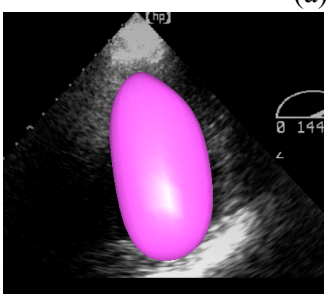

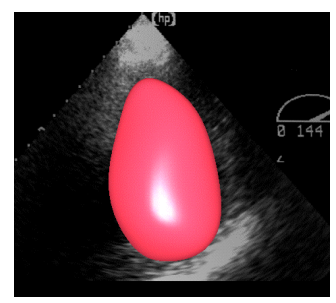

(e)

Fig. 8. Results of the proposed algorithms on real heart data with one image slice overlaid on; (a) (b) 3D endocardical feature points from long-axis window and short-axis window; (c) (e) ICP surface fitting on each window and combined acoustic windows, respectively. 
(left of the 3D points in Figure 8(a)). Using the new method this is filled using information from the parasternal short-axis view (see (b)). Note that, in this experiment, apart from the presence of the shadow in the short-axis window, we just acquired one part of the LV data during the short-axis window scanning.

\section{Conclusions and Future Work}

In this paper we have proposed and performed initially testing on a new method for automatically fusing information from multiple $3 \mathrm{D}$ echocardiographic windows. The approach is novel in the way that it works on the automatically detected feature points and information is fused based on the use of geometric constraints of image acquisition and knowledge of the object. Our experimental results show that fusing information from multiple acoustic windows can fill in data points in shadow regions of other windows and improve the quality of $3 \mathrm{D}$ reconstruction relative to those based on a single acoustic window. Future plans include looking more closely at how the denseness of data affects the method, and evaluating against a reference such as cardiac MR imaging.

Acknowledgments: The authors gratefully acknowledge the financial support of the UK Medical Research Council (grant G9802587), and Dr Nigel Clarke at the Oxford John Radcliffe Hospital for assistance in acquiring experimental data. We would also like to thank Guofang Xiao and David Atkinson for spending time discussing some of this work with us.

\section{References}

1. Giachetti.A. Online analysis of echocardiographic image sequences. Medical Image Analysis, 2(3), 261-284, 1998.

2. Sanchez-Ortiz, G.I., Noble. J.A. et al. Automating LV motion analysis from three dimensional echocardiography. Proceedings of MIUA, Oxford. UK, 85-88, 1999.

3. Sanchez-Ortiz,G.I., Declerck,J. et al. Automating 3D echocardiographic image analysis. Proceedings of MICCAI. Pittsburg, PA, USA, 687-696, 2000.

4. Legget, M.E., Leotta, D.F. et al. System for quantitative 3D echocardiography of the left ventricle based on a magnetic-field position and orientation sensing system. IEEE Trans. Biomedical Engineering, 45(4), 495-504, 1998.

5. Xiao, G.F. 3D freehand ultrasound imaging of breast. PhD thesis, University of Oxford, 2001.

6. Mulet-Parada,M. and Noble,J.A. 2D+T boundary detection in Echocardiography. Proceedings of MICCAI. Cambridge, Mass. U.S.A, 186-196, 1998.

7. Stetten, G.D. and Pizer, S.M. Medial-Node Models to Identify and Measure Objects in RealTime 3D Echocardiography. IEEE Trans. Medical Imaging, 18(10), 1025-1034, 1999.

8. Leotta, D.F. et al. 3D ultrasound imaging of the rotator cuff: spatial compounding and tendon thickness measurement. Ultrasound in Med. \& Biol., 26(4), 509-525, 2000.

9. Besl, P. and McKay, N. A method for registration of 3-D shapes. IEEE Trans. Pattern Analysis and Machine Intelligence, 14(2), 239-256, 1992.

10.Declerck, J. et al. Automatic registration and alignment on a template of cardiac stress and rest reoriented SPECT images. IEEE Trans. Medical Imaging, 16(6), 727-737, 1997. 